\title{
Spinal versus general anesthesia for minimally invasive transforaminal lumbar interbody fusion: implications on operating room time, pain, and ambulation
}

\author{
Gaetano De Biase, MD, ${ }^{1}$ Shaun E. Gruenbaum, MD, PhD, ${ }^{2}$ James L. West, MD, ${ }^{1}$ Selby Chen, MD, ${ }^{1}$ \\ Elird Bojaxhi, MD, ${ }^{2}$ James Kryzanski, MD, ${ }^{3}$ Alfredo Quiñones-Hinojosa, MD, ${ }^{1}$ and \\ Kingsley Abode-lyamah, MD' ${ }^{1}$
}

${ }^{1}$ Department of Neurosurgery, Mayo Clinic, Jacksonville; ${ }^{2}$ Department of Anesthesiology, Mayo Clinic, Jacksonville, Florida; and ${ }^{3}$ Department of Neurosurgery, Tufts Medical Center, Boston, Massachusetts

OBJECTIVE There has been increasing interest in the use of spinal anesthesia (SA) for spine surgery, especially within Enhanced Recovery After Surgery (ERAS) protocols. Despite the wide adoption of SA by the orthopedic practices, it has not gained wide acceptance in lumbar spine surgery. Studies investigating SA versus general anesthesia (GA) in lumbar laminectomy and discectomy have found that SA reduces perioperative costs and leads to a reduction in analgesic use, as well as to shorter anesthesia and surgery time. The aim of this retrospective, case-control study was to compare the perioperative outcomes of patients who underwent minimally invasive surgery (MIS)-transforaminal lumbar interbody fusion (TLIF) after administration of SA with those who underwent MIS-TLIF under GA.

METHODS Overall, 40 consecutive patients who underwent MIS-TLIF by a single surgeon were analyzed; 20 patients received SA and 20 patients received GA. Procedure time, intraoperative adverse events, postoperative adverse events, postoperative length of stay, 3-hour postanesthesia care unit (PACU) numeric rating scale (NRS) pain score, opioid medication, and time to first ambulation were collected for each patient.

RESULTS The two groups were homogeneous for clinical characteristics. A decrease in total operating room (OR) time was found for patients who underwent MIS-TLIF after administration of SA, with a mean OR time of $156.5 \pm 18.9$ minutes versus $213.6 \pm 47.4$ minutes for patients who underwent MIS-TLIF under GA ( $p<0.0001$ ), a reduction of $27 \%$. A decrease in total procedure time was also observed for SA versus GA (122 \pm 16.7 minutes vs $175.2 \pm 10$ minutes; $p$ $<0.0001)$. No significant differences were found in intraoperative and postoperative adverse events. There was a difference in the mean maximum NRS pain score during the first 3 hours in the PACU as patients who received SA reported a lower pain score compared with those who received GA (4.8 \pm 3.5 vs $7.3 \pm 2.7 ; p=0.018)$. No significant difference was observed in morphine equivalents received by the two groups. A difference was also observed in the mean overall NRS pain score, with $2.4 \pm 2.1$ for the SA group versus $4.9 \pm 2.3$ for the GA group $(p=0.001)$. Patients who received SA had a shorter time to first ambulation compared with those who received GA (385.8 \pm 353.8 minutes vs $855.9 \pm 337.4$ minutes; $p$ <0.0001).

CONCLUSIONS The results of this study have pointed to some important observations in this patient population. SA offers unique advantages in comparison with GA for performing MIS-TLIF, including reduced OR time and postoperative pain, and faster postoperative mobilization.

https://thejns.org/doi/abs/10.3171/2021.9.FOCUS21265

KEYWORDS spinal anesthesia; awake MIS-TLIF; awake spine surgery

$\mathrm{T}$ RANSFORAMINAL lumbar interbody fusion (TLIF) is a surgical technique frequently used to address many spinal pathologies. A growing body of literature has shown the advantages of minimally invasive surgery (MIS)-TLIF compared with open TLIF. ${ }^{1-5}$ Recent prog- ress in spine surgery includes the use of the endoscope, robot-assisted screw placement, and navigation guidance. ${ }^{6,7}$ With the goal of improving outcomes and accelerating patient recovery, there has been increasing interest in the use of spinal anesthesia (SA) in spine surgery, particularly for

ABBREVIATIONS ASA = American Society of Anesthesiologists; bpm = beats per minute; EBL = estimated blood loss; ERAS = Enhanced Recovery After Surgery; GA = general anesthesia; $\mathrm{HR}$ = heart rate; MABP = mean arterial blood pressure; $\mathrm{MIS}$ = minimally invasive surgery; NRS = numeric rating scale; OR = operating room; PACU = postanesthesia care unit; SA = spinal anesthesia; TLIF = transforaminal lumbar interbody fusion.

SUBMITTED April 19, 2021. ACCEPTED September 14, 2021.

INCLUDE WHEN CITING DOI: 10.3171/2021.9.FOCUS21265. 
laminectomy and discectomy, especially within Enhanced Recovery After Surgery (ERAS) protocols. ${ }^{8-12}$ Both general anesthesia (GA) and SA have been adopted for surgical treatment of lumbar spine disorders, but GA is used far more widely, and exclusively, in many settings. Regional anesthesia is frequently used in orthopedic surgery and as part of ERAS protocols in other specialties..$^{13}$ Despite the wide adoption of SA by the orthopedic practices for treatment of lower-extremity injuries and total joint arthroplasty, it has not been widely accepted in lumbar spine surgery. The slow adoption of SA for lumbar spine surgery does not appear to be due to scientific or clinical evidence of superiority but, rather, to either surgeon preference and, potentially, lack of exposure to SA, or anesthesiologist preference for GA because of a secured airway prior to placement of the patient in the prone position.

Several studies evaluating SA versus GA in lumbar laminectomy and discectomy have found that SA reduced perioperative costs by reducing drug consumption and led to a reduction in analgesic use, shorter anesthesia and surgery time, reduced blood loss, and less postoperative nausea and vomiting., ${ }^{9,10,14-19}$ To the best of our knowledge, no studies have looked at the perioperative outcomes and potential benefits of SA versus GA in MIS-TLIF.

This retrospective, case-control study was conducted to compare the perioperative outcomes of patients who underwent MIS-TLIF performed by a single surgeon after administration of SA with those who underwent MISTLIF under GA. As long-term outcomes and pain relief are known to be more dependent on patient selection and the effectiveness of the surgical procedure, and less on the anesthesia type, we focused on peri- and intraoperative outcomes to determine the potential benefits and risks of these two anesthetic options.

\section{Methods}

\section{Study Design and Patient Population}

After approval of the study protocol by the IRB, data were reviewed by an independent observer for all patients who underwent MIS-TLIF by a single surgeon, the senior author (K.A.I.), at our institution between July 2019 and September 2020. Information was stripped of identifiers, and all critical identifiers were masked as data were acquired. The patients selected for this study were adults who underwent either one- or two-level MIS-TLIF. To limit confounding variables, patients undergoing revision surgery were excluded.

This retrospective case-control study analyzed 40 opioid-naive patients who underwent MIS-TLIF for lumbar degenerative disc disease, spinal stenosis, or grade I or II degenerative spondylolisthesis. We looked at 20 consecutive patients who underwent MIS-TLIF after induction of SA by a single surgeon (K.A.I.) from February 2020 to September 2020 (Fig. 1). At our institution, we started performing MIS-TLIF with SA in February 2020 and routinely offered it to patients unless they met any of the following exclusion criteria: history of seizure or intracranial hypertension, coagulopathy, infection at site of needling, hypovolemia, severe spinal stenosis, myelographic demonstration of arachnoiditis, claustrophobia, or severe res-
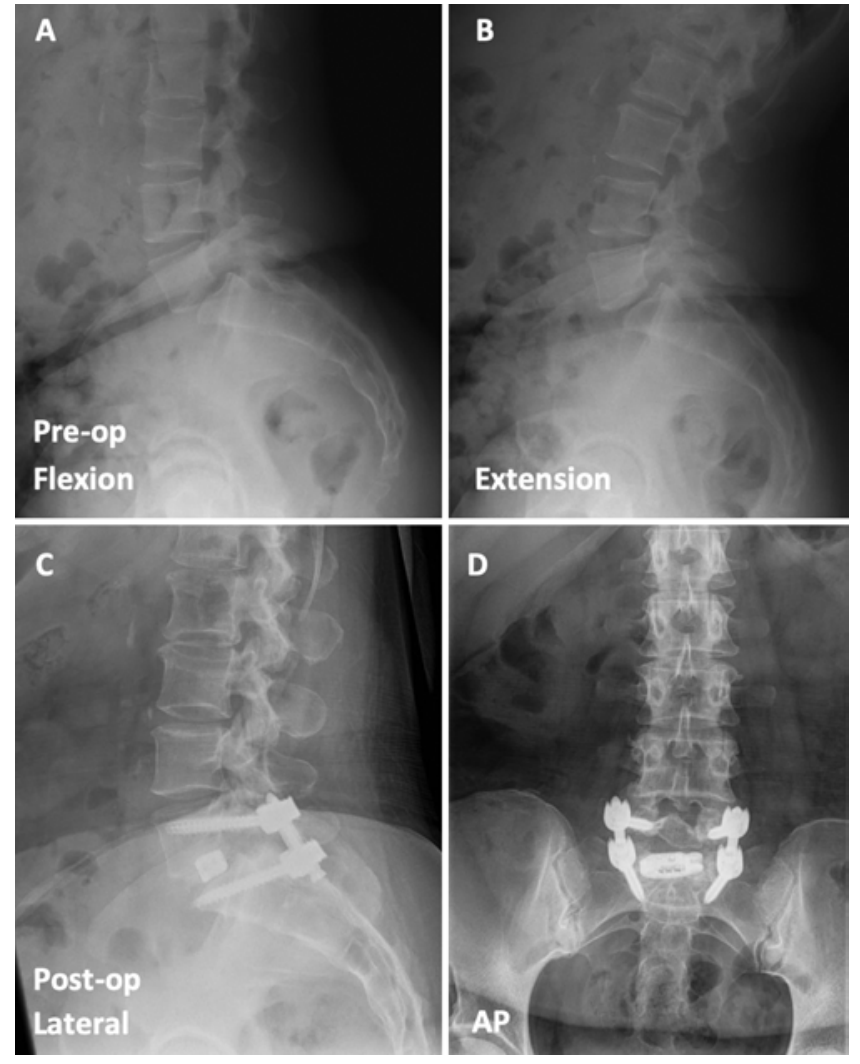

FIG. 1. Images obtained in a 51-year-old female with L5 left leg pain distribution and L5-S1 spondylolisthesis. After failure of conservative management (physical therapy, injections, and gabapentin), the patient underwent an L5-S1 MIS-TLIF with SA. A and B: Preoperative flexion (A) and extension (B) lumbar radiographs demonstrating bilateral L5 pars defects with grade I anterolisthesis of L5-S1, with no evidence of dynamic instability. C and D: Lateral (C) and anteroposterior (AP; D) lumbar radiographs obtained 9 months postoperatively, demonstrating good positioning of the L5-S1 pedicle screws and intervertebral cage.

piratory problems. Patients were also offered the option to undergo the procedure under GA. The control group consisted of 20 consecutive patients who underwent MISTLIF under GA by the same surgeon from July 2019 to February 2020, before we adopted SA for MIS-TLIF. To minimize selection bias, the charts of the GA group were reviewed by an anesthesiologist to ensure that they did not meet any of the exclusion criteria for SA, confirming that they would have been potential candidates for SA. The same surgical technique was used for all patients. Our group has previously described the technique we use to perform MIS-TLIF with SA using a pedicle-based retractor system ${ }^{12}$ which is the same technique used for patients who received GA in this study. The groups were checked for clinical factors including comorbidities (obesity, hypertension, diabetes, and obstructive sleep apnea), age, and American Society of Anesthesiologists (ASA) class, to verify that they were homogeneous. The anesthetic protocols were established by members of the anesthesia department and were maintained without variation during the study period. Prior to surgery, the anesthesiologist reviewed all medical records and confirmed the medical, surgical, and anesthesia history with the patient and sur- 
geon to determine the most appropriate anesthesia plan. The specific anesthetic drugs were chosen at the discretion of the anesthesiologist, and all patients were monitored with standard ASA monitoring regardless of anesthetic technique.

\section{GA Protocol}

For patients in the GA group, premedication with intravenous midazolam (1-2 mg) and/or fentanyl (25-150 $\mu \mathrm{g})$ was commonly administered. After preoxygenation with $100 \%$ oxygen, GA was induced with a combination of intravenous propofol $(1.5-2.0 \mathrm{mg} / \mathrm{kg})$ with or without lidocaine $(20-100 \mathrm{mg})$ and fentanyl $(50-250 \mu \mathrm{g})$. Endotracheal intubation was performed via direct or video laryngoscopy after achieving adequate muscle relaxation with intravenous rocuronium $(0.6-1.2 \mathrm{mg} / \mathrm{kg})$ or succinylcholine $(1 \mathrm{mg} / \mathrm{kg})$. Patients were positioned prone, and the head was maintained in a foam pillow. GA was typically maintained with propofol (75-200 $\mathrm{mg} / \mathrm{kg}$ per minute) or sevoflurane (1.0\% to $2.0 \%$ in $40 \%$ to $70 \%$ oxygen). At the completion of the procedure, the patient was repositioned supine, and the anesthetic was discontinued. The trachea was extubated after adequate reversal of nondepolarizing muscle relaxant, as indicated, and after the patient was awake, breathing spontaneously, and following commands.

\section{SA Protocol}

For patients in the SA group, premedication with intravenous midazolam (1-2 $\mathrm{mg}$ ) and/or fentanyl (25-150 $\mu \mathrm{g})$ was commonly administered. In the operating room (OR), patients were positioned seated and bent slightly forward, and their back was cleaned with topical chlorhexidine solution. After application of subcutaneous local anesthesia with $2 \%$ lidocaine $(0.5-2 \mathrm{~mL})$, a spinal needle was introduced between the L3-4 or L4-5 vertebral space under sterile conditions (1 level above or below the location of the planned surgery, which was confirmed with the surgeon). Confirmation that the tip of the needle was in the subarachnoid space was made with the presence of freeflowing cerebrospinal fluid. Isobaric bupivicaine $(0.5 \%$, $2.5 \mathrm{~mL}$ ) was slowly injected in the subarachnoid space. The patient was then positioned prone on a Wilson frame, and the head was placed in a foam pillow with adequate ventilation to prevent rebreathing of carbon dioxide. Light sedation was administered and included intravenous dexmedetomidine $(0.2-0.7 \mu \mathrm{g} / \mathrm{kg}$ per hour) and/or propofol $(10-50 \mu \mathrm{g} / \mathrm{kg}$ per minute). Surgery proceeded after confirmation of an adequate surgical anesthetic level. For patients undergoing surgery after administration of SA we do not routinely place a Foley catheter. At the completion of the procedure, the patient was repositioned supine, and the anesthetic was discontinued. All patients received standardized perioperative multimodal analgesia (an oral acetaminophen regimen of $1000 \mathrm{mg}$ every 6 hours that was started in the preoperative holding area) and nausea and vomiting prophylaxis (ondansetron $4 \mathrm{mg}$ intravenously prior to emergence). Postoperatively, patients received hydrocodone-acetaminophen (10-325 mg) as needed for pain control every 4 hours; for severe pain, some patients received hydromorphone ( $0.2 \mathrm{mg}$ intravenously). Patients were mobilized once the spinal block wore off and monitored for urinary retention. Chan et al. have reported on the safe and effective use of off-label liposomal bupivacaine injections to each incision/percutaneous screw track to provide long-acting local anesthesia. ${ }^{20}$ In our series, per the surgeon's preference, we did not use liposomal bupivacaine, opting for regular local anesthetic instead.

\section{Perioperative Outcomes}

For each patient, data were collected from the electronic medical record system including the total OR time (from patient entering the OR to patient leaving the OR, which included administration of the SA for the SA group), total procedure time (from skin incision to placement of the surgical dressing), time to leave the OR (from placement of the surgical dressing to the patient leaving the OR), intraoperative adverse events, intraoperative estimated blood loss (EBL), postoperative adverse events, and postoperative length of stay. We also recorded all the pain medications that patients received during the first 3 hours in the postanesthesia care unit (PACU), as well as all numeric rating scale (NRS) pain scores $(0-10)$ during the first 3 hours in the PACU. To analyze the postoperative narcotic requirement, an online calculator (https:// clincalc.com/opioids) was used by an anesthesiologist to calculate the conversions of opioid medications to morphine equianalgesic doses. The equianalgesic conversions used in this calculator are based on the American Pain Society guidelines and critical review papers. ${ }^{21,22}$ The time to first ambulation was also collected, calculated from the time patients left the OR to the first ambulation. Patient complaints of nausea, or actual episodes of vomiting, during the PACU admission were recorded in the chart.

\section{Perioperative Analgesic and Antiemetic Regimen}

In both groups, patients received preoperative multimodal analgesics before arrival to the OR which included oral acetaminophen $(1000 \mathrm{mg})$, celecoxib $(200 \mathrm{mg})$, and oxycodone (10 mg). Intraoperatively, nausea and vomiting prophylaxis included intravenously administered dexamethasone $(10 \mathrm{mg})$ and ondansetron $(4 \mathrm{mg})$. Postoperatively, patients received oral oxycodone $(5 \mathrm{mg}-10 \mathrm{mg}$ ) for moderate pain and intravenously administered hydromorphone $(0.2 \mathrm{mg}-0.5 \mathrm{mg})$ for severe pain.

\section{Intraoperative Hemodynamic Parameters}

We also collected intraoperative hemodynamic parameters including the mean heart rate (HR) and mean arterial blood pressure (MABP), which were recorded at the time the patient first entered the OR and subsequently monitored every minute for the HR and every 5 minutes for the MABP.

\section{Statistical Analysis}

Descriptive demographic statistics were calculated, using the mean as a measure of central tendency and the standard deviation as a measure of dispersion. The chisquare test was used to compare categorical variables and comparisons of continuous variables were made using the 
TABLE 1. Patient characteristics stratified by anesthesia type

\begin{tabular}{|c|c|c|c|}
\hline Variable & $S A(n=20)$ & $\mathrm{GA}(\mathrm{n}=20)$ & $\mathrm{p}$ Value \\
\hline \multicolumn{4}{|l|}{ Sex } \\
\hline M & $9(45)$ & $10(50)$ & 0.751 \\
\hline $\mathrm{F}$ & $11(55)$ & $10(50)$ & \\
\hline Mean age, yrs & $63.5 \pm 10.3$ & $62.3 \pm 11.3$ & 0.364 \\
\hline Mean BMI & $30.7 \pm 5.2$ & $30.6 \pm 6.4$ & 0.925 \\
\hline Obesity & $9(45)$ & $7(35)$ & 0.518 \\
\hline DM2 & $4(20)$ & $3(15)$ & 0.677 \\
\hline HTN & $10(50)$ & $15(75)$ & 0.102 \\
\hline OSA & $4(20)$ & $2(10)$ & 0.375 \\
\hline \multicolumn{4}{|l|}{ Operated levels } \\
\hline Single & $17(85)$ & $17(85)$ & $>0.99$ \\
\hline Double & $3(15)$ & $3(15)$ & \\
\hline \multicolumn{4}{|l|}{ ASA class } \\
\hline II & $11(55)$ & $8(40)$ & 0.342 \\
\hline III & $9(45)$ & $12(60)$ & \\
\hline Mean preop HR, bpm & $75 \pm 10.4$ & $79.3 \pm 11$ & 0.217 \\
\hline Mean preop MABP, mm Hg & $105.9 \pm 18$ & $96.9 \pm 11.5$ & 0.067 \\
\hline
\end{tabular}

Student t-test. All tests were performed at a significance level of 0.05. All analyses were performed using IBM SPSS Statistics version 24 (IBM Corp.).

\section{Results}

Overall, 20 patients underwent a primary MIS-TLIF after administration of SA from February 2020 to September 2020, and 20 patients underwent a primary MISTLIF under GA from July 2019 to February 2020; all surgeries were performed by the senior author (K.A.I.).

\section{Patient Characteristics}

Between the SA and GA groups, there was no statistically significant difference in age; sex; BMI; prevalence of diabetes, hypertension, and obstructive sleep apnea; ASA physical status classification; or number of operated levels (Table 1). The patients who underwent MIS-TLIF after administration of SA ranged in age from 50 to 84 years, with a mean of 63.5 years (SD 10.3 years), while the GA group ranged in age from 43 to 80 years with a mean of 62.3 years (SD 11.3 years) $(p=0.364)$. The mean BMI was 30.7 \pm 5.2 for the SA group and $30.6 \pm 6.4$ for the GA group $(\mathrm{p}$ $=0.925$ ). The mean ASA class for the SA and GA groups was $2.5 \pm 0.5$ and $2.6 \pm 0.5$, respectively $(\mathrm{p}=0.355) ; 11$ patients $(55 \%)$ were in class II and 9 patients $(45 \%)$ were in class III in the SA group, while 8 patients $(40 \%)$ were in class II and 12 patients (60\%) were in class III in the GA group $(\mathrm{p}=0.342)$. There was no difference in the number of operated levels; both groups had 17 patients who underwent a single-level MIS-TLIF and 3 patients who underwent a two-level MIS-TLIF ( $p>0.99$ ). After review by an anesthesiologist, none of the charts of patients in the GA
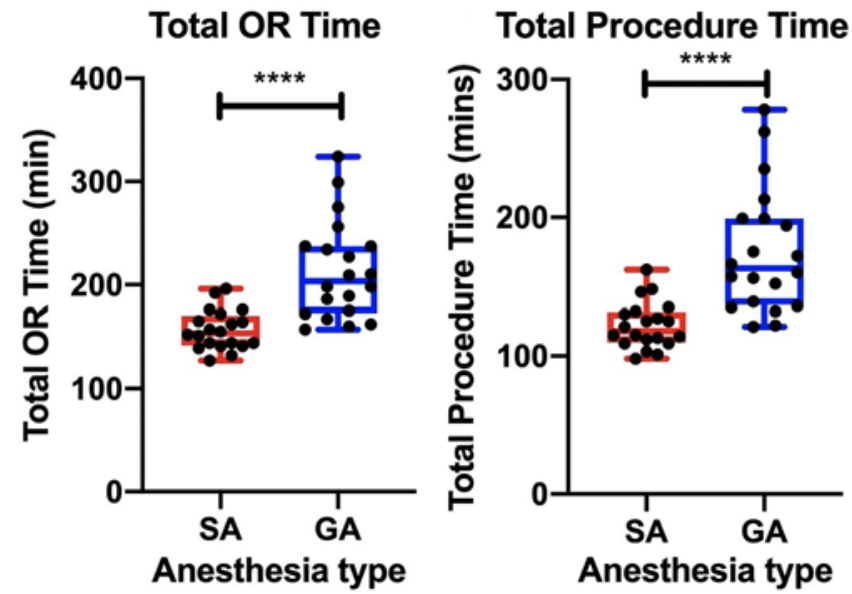

FIG. 2. Comparison of total OR time and total procedure time for patients who underwent MIS-TLIF with SA versus GA. The black dots indicate the specific values for each patient. ${ }^{* * *} p \leq 0.0001$.

group fit any of the exclusion criteria for SA, indicating that they could have been potential SA candidates. There were no failures of anesthesia in this series, so that no patients required conversion from SA to GA. Surgery was successfully completed in each anesthesia treatment group without any intraoperative adverse events, and no intraoperative CSF leaks or airway complications were encountered. No patients developed postoperative urinary retention requiring Foley catheter placement or straight bladder catheterizations. The only postoperative complication occurred in a patient who underwent surgery with SA and developed postoperative ileus, which resolved with laxatives.

\section{Intraoperative Variables: OR Time and Procedure Time}

We observed a significant decrease in the total OR time for patients who underwent MIS-TLIF after induction of $\mathrm{SA}$, with a mean total OR time of $156.5 \pm 18.9$ minutes versus $213.6 \pm 47.4$ minutes for the GA group $(\mathrm{p}<0.0001)$. This corresponds to a mean reduction in OR time by $27 \%$ or 57 minutes for the SA group. We also observed a significant reduction in the total procedure time: $122 \pm 16.7$ minutes for patients who received SA versus $175.2 \pm 10$ minutes ( $\mathrm{p}<0.0001)$ for those who received GA, a $30 \%$ reduction or 53 minutes less (Fig. 2). A significant reduction was also observed in the time from the procedure end to the patient leaving the OR in the SA group (3.25 \pm 1.78 minutes vs $9.7 \pm 7.1$ minutes; $p<0.0001$ ). We did not observe a significant difference between the two groups in the time from when the patient entered the OR to the procedure start (SA $34.5 \pm 8.2$ minutes vs GA $38.5 \pm 6.5$ minutes; $\mathrm{p}=0.102$ ) (Table 2).

\section{Hemodynamic Parameters}

There was no statistically significant difference in the mean EBL for the SA group $(39 \pm 30.2 \mathrm{~mL})$ compared with the GA group $(57.8 \pm 42.1 \mathrm{~mL})(\mathrm{p}=0.114)$. No statistically significant difference was observed in the mean intraoperative MABP, which was $80.8 \pm 8.1 \mathrm{~mm} \mathrm{Hg}$ for the SA group and $84.4 \pm 9.6 \mathrm{~mm} \mathrm{Hg}$ for the GA group (p $=0.212$ ). However, we observed a significant difference in 
TABLE 2. Intraoperative parameters

\begin{tabular}{lccc}
\hline \multirow{2}{*}{ Variable } & \multicolumn{2}{c}{ Mean \pm SD } & \\
\cline { 2 - 3 } & $34.5 \pm 8.2$ & $38.5 \pm 6.5$ & 0.102 \\
\hline $\begin{array}{l}\text { Time from entering OR } \\
\text { to op start, mins }\end{array}$ & $122 \pm 16.7$ & $175.2 \pm 10$ & $<0.0001$ \\
\hline Total op time, mins & $156.5 \pm 18.9$ & $213.6 \pm 47.4$ & $<0.0001$ \\
\hline Total OR time, mins & $3.25 \pm 1.78$ & $9.7 \pm 7.1$ & $<0.0001$ \\
\hline Time to leave OR, mins & $39 \pm 30.2$ & $57.8 \pm 42.1$ & 0.114 \\
\hline EBL, mL & $80.8 \pm 8.1$ & $84.4 \pm 9.6$ & 0.212 \\
\hline Intraop MABP, mm Hg & $66 \pm 7.7$ & $73.1 \pm 7.6$ & 0.006 \\
\hline Intraop HR, bpm & &
\end{tabular}

Boldface type indicates statistical significance.

the mean intraoperative HR, with the SA group having 66 \pm 7.7 beats per minute (bpm) versus $73.1 \pm 7.6 \mathrm{bpm}$ for the GA group $(\mathrm{p}=0.006)$.

\section{Perioperative Findings: Pain Scores and Ambulation}

There was a significant difference in the mean maximum NRS pain score during the first 3 hours in the PACU, with $4.8 \pm 3.5$ for the SA group versus $7.3 \pm 2.7$ for the GA group $(\mathrm{p}=0.018)$. A significant difference was also observed in the mean overall NRS pain score during the first 3 hours in the PACU, with $2.4 \pm 2.1$ for the SA group versus $4.9 \pm 2.3$ for the GA group $(p=0.001)$. No significant difference was observed between the groups in total morphine equivalents received during the first 3 hours in the PACU $(3.71 \pm 4.2 \mathrm{mg}$ vs $5.91 \pm 6.7 \mathrm{mg}$ of morphine sulfate; $p=0.22$ ) (Fig. 3). No significant difference was observed in the mean time to administration of the first postoperative pain medication, with $53 \pm 37.9$ minutes for the SA group versus $55.2 \pm 49.3$ minutes for the GA group $(\mathrm{p}=0.885)$. No cases of postoperative vomiting were recorded. A significant difference was observed in the mean time to first ambulation, with the SA group ambulating $385.8 \pm 353.8$ minutes after leaving the OR versus 855.9 \pm 337.4 minutes for the GA group $(\mathrm{p}<0.0001)$ (Table 3$)$.

\section{Discussion}

SA is gaining popularity as an alternative to GA for spine surgery. Only one randomized prospective study has been conducted to investigate whether either of these techniques is superior to the other, and that study included only lumbar discectomies and laminectomies. ${ }^{23}$ The present case-controlled study, though retrospective, represents the first single-surgeon series comparing SA with GA for MIS-TLIF. Our study validates what was also observed for lumbar laminectomies and discectomies, that SA for MIS-TLIF offers some unique advantages. Wang et al. have reported the efficacy and safety of endoscopic TLIF without GA, using expandable bone grafts and percutaneous fixation under local anesthesia to avoid GA. ${ }^{24,25}$ The mean operative time in their study was $84.5 \pm 21.7$ minutes for single-level fusions and $128.1 \pm 48.6$ minutes for two-level fusions. In our series, we used a direct decompression technique performed through a pedicle-based retraction system, as previously reported..$^{12}$
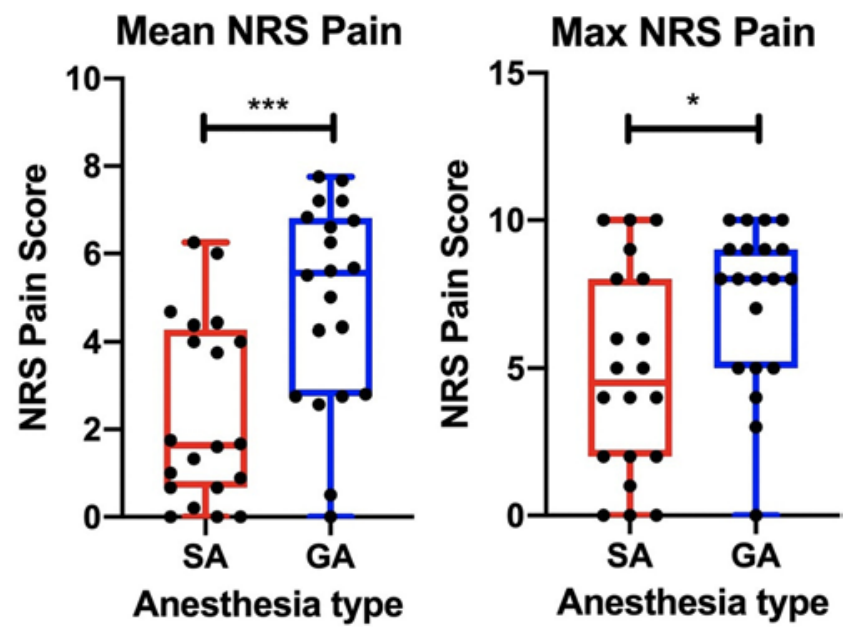

FIG. 3. Comparison of the mean maximum and mean overall NRS pain scores during the first 3 hours in the PACU after MIS-TLIF for patients who received $\mathrm{SA}$ versus those who received $\mathrm{GA}$. The black dots indicate the specific values for each patient. ${ }^{*} p \leq 0.05,{ }^{* *} p \leq 0.001$.

Despite the retrospective nature of the study, we minimized confounding variables by analyzing cases from a single surgeon at a single institution and managed by the same anesthesia staff. Furthermore, to minimize selection bias, the GA control group was selected from the few months prior to our institution starting an SA protocol for spine surgery; therefore, those patients received GA independently from whether or not they were deemed ideal candidates for SA. Additionally, their charts were reviewed by an anesthesiologist to ensure that they did not meet any of the SA exclusion criteria, confirming that they would have been potential candidates for SA.

\section{Implications on OR Times}

SA significantly reduced both the total OR time and total procedure time, with no events of failed intraoperative anesthesia. We observed a $27 \%$ reduction in the total OR time for the SA group, which is explained by the reduced time required at the end of the procedure for patient emergence from anesthesia (GA) and assessment of their

TABLE 3. Postoperative outcomes

\begin{tabular}{|c|c|c|c|}
\hline \multirow[b]{2}{*}{ Variable } & \multicolumn{2}{|c|}{ Mean \pm SD } & \multirow[b]{2}{*}{ p Value } \\
\hline & SA & GA & \\
\hline $\begin{array}{l}\text { Overall 3-hr PACU NRS } \\
\text { score }\end{array}$ & $2.4 \pm 2.1$ & $4.9 \pm 2.3$ & 0.001 \\
\hline Max 3-hr PACU NRS score & $4.8 \pm 3.5$ & $7.3 \pm 2.7$ & 0.018 \\
\hline $\begin{array}{l}\text { 3-hr PACU opioid require- } \\
\text { ment, mg/MS }\end{array}$ & $3.71 \pm 4.2$ & $5.91 \pm 6.7$ & 0.22 \\
\hline $\begin{array}{l}\text { Time to 1st pain medica- } \\
\text { tion, mins }\end{array}$ & $53 \pm 37.9$ & $55.2 \pm 49.3$ & 0.885 \\
\hline Time to ambulation, mins & $385.8 \pm 353.8$ & $855.9 \pm 337.4$ & $<0.0001$ \\
\hline Time to discharge, hrs & $32.8 \pm 31.7$ & $39.3 \pm 18.9$ & 0.435 \\
\hline
\end{tabular}

$\mathrm{mg} / \mathrm{MS}=$ milligrams of morphine sulfate.

Boldface type indicates statistical significance. 
responsiveness and respiratory function before extubation, as well as a significant decrease in the procedure time.

Reduced bleeding due to the different hemodynamic characteristics has been suggested as a factor that may play a role in decreased procedure times in patients undergoing spine surgery with SA. The difference in procedure times cannot be explained by increased experience with performing MIS-TLIF, as the cases were only a few months apart and the senior author had already reached the plateau of the learning curve for MIS-TLIF before the cases included in the current study.

\section{Hemodynamic Parameters}

Previous studies have reported reduced blood loss in patients undergoing spine surgery with SA, potentially explained by reduced intrathoracic pressures when patients are allowed to breathe spontaneously, in combination with a sympathetic blockade. In our series, we did not observe a significant difference in EBL, given the already small amount resulting from a minimally invasive technique. Multiple studies have shown that HR and blood pressure are lower with SA than with GA., ${ }^{9,26,27}$ Other studies have not found a difference between the two methods. ${ }^{28} \mathrm{We}$ observed a significant difference in the mean intraoperative HR but no significant difference in the intraoperative MABP. According to current literature, the improved hemodynamic stability of SA over GA might be due to an inhibited release of stress hormones intraoperatively, leading to lower elevation and fluctuations of MABP and HR. ${ }^{23,29}$

\section{Implications on Postoperative Pain and Ambulation}

Since in some cases the PACU length of stay was prolonged due to a lack of bed availability in the hospital and some SA patients who were discharged on the same day as the procedure stayed in the PACU until discharge, we did not compare total PACU time. Patients who underwent MIS-TLIF after administration of SA experienced less postoperative pain, having a lower mean overall and mean maximum NRS pain score in the first 3 hours in the PACU compared with patients who underwent MIS-TLIF under GA. No difference in narcotic requirements were noted between the groups, indicating that, with the same dosage of narcotic medications, patients who underwent the procedure with SA had better pain control. The lack of significant difference in opioid use in the presence of a significantly lower pain score could also be explained by the small sample size and potential differences in pain tolerance between the groups, which we could not account for given the nonrandomized nature of the study. Improved postoperative comfort has been shown to be linked to higher levels of patient satisfaction. We also observed a significant and meaningful decrease in the time to first ambulation as patients who underwent MIS-TLIF with SA ambulated an average 7.8 hours before those who underwent surgery under GA, indicating the potential for faster recovery and reduced complications.

We did not observe a significant difference in length of stay between the two groups, with most patients being discharged home the 1 st and 2 nd day after surgery. None of the patients experienced nausea, emesis, or urinary retention requiring catheterization. The only postoperative complication occurred in a patient who underwent surgery with SA and developed postoperative ileus, which resolved with laxatives.

\section{Limitations and Future Perspectives}

Limitations of the study are related to its retrospective nature. Although every effort was made to ensure that the case complexity was comparable, there is a possibility for selection bias, as the anesthesia technique was not randomized. The cases were consecutive and performed by a single surgeon and with the same anesthesia team. An independent observer reviewed operative records to establish that the case complexity was comparable between the groups. All patients with medical conditions that would have influenced selection of anesthesia method were excluded from the study. Although many of the flaws frequently affecting retrospective studies were minimized, we recognize that some selection bias and potential inaccuracies in the charts may exist. We believe that these biases, if present, are not significant enough to negate our conclusions. Future prospective randomized controlled trials are indicated to validate our results. Another limitation is the small sample size which did not allow us to observe any differences in terms of intra- or postoperative complications; thus, we focused mainly on the perioperative outcomes.

This study represents the first single-surgeon series comparing SA versus GA for MIS-TLIF. The aim of the study was to compare the perioperative outcomes of patients who underwent MIS-TLIF after administration of SA with those who underwent surgery under GA. We found SA to be superior to GA in some aspects; patients who underwent MIS-TLIF with SA experienced less postoperative pain and were able to ambulate much faster, and SA was also associated with a decreased OR time.

\section{Conclusions}

Our study demonstrates that SA is effective for performing MIS-TLIF, offering unique advantages compared with GA, including reduced postoperative pain, reduced OR time, and faster postoperative mobilization. Strong communication between the neurosurgeon and the anesthesia team is key, and our findings indicate the potential for increased adoption of SA for lumbar spine fusion.

\section{Acknowledgments}

We thank Cathleen and Bill Stender for their support.

\section{References}

1. Parker SL, Mendenhall SK, Shau DN, Zuckerman SL, Godil SS, Cheng JS, McGirt MJ. Minimally invasive versus open transforaminal lumbar interbody fusion for degenerative spondylolisthesis: comparative effectiveness and cost-utility analysis. World Neurosurg. 2014;82(1-2):230-238.

2. Parker SL, Adogwa O, Bydon A, Cheng J, McGirt MJ. Costeffectiveness of minimally invasive versus open transforaminal lumbar interbody fusion for degenerative spondylolisthesis associated low-back and leg pain over two years. World Neurosurg. 2012;78(1-2):178-184.

3. Mobbs RJ, Phan K, Malham G, Seex K, Rao PJ. Lumbar 
interbody fusion: techniques, indications and comparison of interbody fusion options including PLIF, TLIF, MI-TLIF, OLIF/ATP, LLIF and ALIF. J Spine Surg. 2015;1(1):2-18.

4. Phan K, Rao PJ, Kam AC, Mobbs RJ. Minimally invasive versus open transforaminal lumbar interbody fusion for treatment of degenerative lumbar disease: systematic review and meta-analysis. Eur Spine J. 2015;24(5):1017-1030.

5. Yoon JW, Wanderman NR, Kerezoudis P, Alvi MA, De Biase $\mathrm{G}$, Akinduro OO, et al. Enterobacter infection after spine surgery: an institutional experience. World Neurosurg. 2019; 123:e330-e337.

6. De Biase G, Gassie K, Garcia D, Abode-Iyamah K, Deen G, Nottmeier E, Chen S. Perioperative comparison of roboticassisted versus fluoroscopically guided minimally invasive transforaminal lumbar interbody fusion. World Neurosurg. 2021;149:e570-e575

7. De Biase G, Chen S, Akinduro O, Quinones-Hinojosa A, Abode-Iyamah K. Awake robotic minimally-invasive L4-5 transforaminal lumbar: interbody fusion (TLIF). World Neurosurg. 2021;148:93.

8. Liu B, Liu S, Wang Y, Zhao B, Zhao T, Zhao L, et al. Neurosurgical enhanced recovery after surgery (ERAS) programme for elective craniotomies: are patients satisfied with their experiences? A quantitative and qualitative analysis. BMJ Open. 2019;9(11):e028706.

9. Agarwal P, Pierce J, Welch WC. Cost Analysis of spinal versus general anesthesia for lumbar diskectomy and laminectomy spine surgery. World Neurosurg. 2016;89:266-271.

10. Deng H, Coumans JV, Anderson R, Houle TT, Peterfreund RA. Spinal anesthesia for lumbar spine surgery correlates with fewer total medications and less frequent use of vasoactive agents: a single center experience. PLoS One. 2019;14(6): e0217939.

11. De Biase G, Chen S, Bydon M, Elder BD, McClendon J, Deen HG, et al. Postoperative restrictions after anterior cervical discectomy and fusion. Cureus. 2020;12(8):e9532.

12. De Biase G, Bechtle P, Leone B, Quinones-Hinojosa A, Abode-Iyamah K. Awake minimally invasive transforaminal lumbar interbody fusion with a pedicle-based retraction system. Clin Neurol Neurosurg. 2021;200:106313.

13. Donauer K, Bomberg H, Wagenpfeil S, Volk T, Meissner W, Wolf A. Regional vs. general anesthesia for total knee and hip replacement: an analysis of postoperative pain perception from the international PAIN OUT registry. Pain Pract. 2018; 18(8):1036-1047.

14. Lessing NL, Edwards CC II, Brown CH IV, Ledford EC, Dean CL, Lin C, Edwards CC. Spinal anesthesia in elderly patients undergoing lumbar spine surgery. Orthopedics. 2017; 40(2):e317-e322.

15. Walcott BP, Khanna A, Yanamadala V, Coumans JV, Peterfreund RA. Cost analysis of spinal and general anesthesia for the surgical treatment of lumbar spondylosis. J Clin Neurosci. 2015;22(3):539-543.

16. Vural C, Yorukoglu D. Comparison of patient satisfaction and cost in spinal and general anesthesia for lumbar disc surgery. Turk Neurosurg. 2014;24(3):380-384.

17. Meng T, Zhong Z, Meng L. Impact of spinal anaesthesia vs. general anaesthesia on peri-operative outcome in lumbar spine surgery: a systematic review and meta-analysis of randomised, controlled trials. Anaesthesia. 2017;72(3):391-401.

18. McLain RF, Kalfas I, Bell GR, Tetzlaff JE, Yoon HJ, Rana M. Comparison of spinal and general anesthesia in lumbar laminectomy surgery: a case-controlled analysis of 400 patients. J Neurosurg Spine. 2005;2(1):17-22.

19. Lessing NL, Edwards CC II, Lin C, Brown CH IV. Complex lumbar spine fusion for an elderly patient under spinal anesthesia. Orthopedics. 2017;40(5):e915-e917.
20. Chan AKH, Choy W, Miller CA, Robinson LC, Mummaneni PV. A novel technique for awake, minimally invasive transforaminal lumbar interbody fusion: technical note. Neurosurg Focus. 2019;46(4):E16.

21. Principles of analgesic use in the treatment of acute pain and chronic cancer pain, 2nd edition. American Pain Society. Clin Pharm. 1990;9(8):601-612.

22. Anderson R, Saiers JH, Abram S, Schlicht C. Accuracy in equianalgesic dosing. conversion dilemmas. J Pain Symptom Manage. 2001;21(5):397-406.

23. Attari MA, Mirhosseini SA, Honarmand A, Safavi MR. Spinal anesthesia versus general anesthesia for elective lumbar spine surgery: a randomized clinical trial. J Res Med Sci. 2011;16(4):524-529.

24. Wang MY, Grossman J. Endoscopic minimally invasive transforaminal interbody fusion without general anesthesia: initial clinical experience with 1-year follow-up. Neurosurg Focus. 2016;40(2):E13.

25. Kolcun JPG, Brusko GD, Basil GW, Epstein R, Wang MY. Endoscopic transforaminal lumbar interbody fusion without general anesthesia: operative and clinical outcomes in 100 consecutive patients with a minimum 1-year follow-up. Neurosurg Focus. 2019;46(4):E14.

26. Davis FM, Laurenson VG, Lewis J, Wells JE, Gillespie WJ. Metabolic response to total hip arthroplasty under hypobaric subarachnoid or general anaesthesia. Br J Anaesth. 1987; 59(6):725-729.

27. Edgcombe H, Carter K, Yarrow S. Anaesthesia in the prone position. Br J Anaesth. 2008;100(2):165-183.

28. Sadrolsadat SH, Mahdavi AR, Moharari RS, Khajavi MR, Khashayar P, Najafi A, Amirjamshidi A. A prospective randomized trial comparing the technique of spinal and general anesthesia for lumbar disk surgery: a study of 100 cases. Surg Neurol. 2009;71(1):60-65.

29. Basaranoglu G, Erkalp K, Saidoglu L, Aydas D, Ozdemir $\mathrm{H}$, Teker M. Selective spinal anesthesia for limb amputation above knee level. J Clin Anesth. 2011;23(2):169.

\section{Disclosures}

The authors report no conflict of interest concerning the materials or methods used in this study or the findings specified in this paper.

\section{Author Contributions}

Conception and design: Abode-Iyamah, De Biase, Gruenbaum, Chen, Quiñones-Hinojosa. Acquisition of data: De Biase, West. Analysis and interpretation of data: Abode-Iyamah, De Biase, Gruenbaum, West, Bojaxhi. Drafting the article: Abode-Iyamah, De Biase, Chen. Critically revising the article: all authors. Reviewed submitted version of manuscript: all authors. Approved the final version of the manuscript on behalf of all authors: Abode-Iyamah. Statistical analysis: De Biase. Administrative/ technical/material support: Abode-Iyamah, Chen, Bojaxhi, Quiñones-Hinojosa. Study supervision: Abode-Iyamah, De Biase, Quiñones-Hinojosa.

\section{Supplemental Information \\ Videos}

Video Abstract. https://vimeo.com/642396196.

\section{Correspondence}

Kingsley Abode-Iyamah: Mayo Clinic, Jacksonville, FL. abode-iyamah.kingsley@mayo.edu. 